\title{
NUMERICAL INVESTIGATION OF OLEO-PNEUMATIC SHOCK ABSORBER: SETUP AND VALIDATION
}

\author{
Ahmed A. Sheikh Al-Shabab ${ }^{1}$, Dimitrios Vitlaris ${ }^{1}$, Zhonglu Lin ${ }^{1}$, Bojan Grenko ${ }^{1}$, \\ Panagiotis Tsoutsanis $^{1}$, Antonis Antoniadis ${ }^{1}$ and Martin Skote ${ }^{1}$ \\ ${ }^{1}$ School of Aerospace, Transport and Manufacturing, Cranfield University, \\ College Road, Cranfield, Bedfordshire, MK43 0AL, UK \\ Corresponding Author: A.A.Sheikh-Al-Shabab@Cranfield.ac.uk
}

Key words: Shock Absorber, Multi-Physics, CFD, OpenFOAM

\begin{abstract}
The simulation of an oleo-pneumatic shock absorber is discussed focusing on the solver validation and high fidelity case setup. The multi-physics nature of the problem is tackled by conducting a range of validation cases in the base areas expected to be of relevance. A dynamic system model of the shock absorber is used to generate physically consistent boundary conditions. In addition, steady RANS simulations provide a preliminary insight into the internal flow development and to assist in the design of higher resolution grids.
\end{abstract}

\section{INTRODUCTION}

Aircraft Landing Gear Shock Absorbers (LGSA) are used to reduce the loads on the aircraft structure during the main landing impact and to provide a more comfortable journey for the payload transported inside the aircraft during ground manoeuvring [1]. Most modern aircraft use an oleo-pneumatic shock absorber, however, despite being a relatively stable component on aircraft for decades, there is relatively little research on the internal dynamics of shock absorbers in the public domain literature. This literature gap is partly a reflection of the challenging nature of the problem, as it is an unsteady system involving turbulence, multiphase flow, compressibility, heat transfer and a complex geometry.

Focusing on the computational side of LGSA research and considering the difficulties described above with regards to the multi-physics unsteady nature of the internal shock absorber flow field, the aim of the present work is to discuss the validation process of a selected numerical flow field solver and to consider the setup of LGSA simulations.

\section{Methodology}

The scarcity of data about the internal shock absorber field makes it difficult to ascertain the important physics to model at a given stage of the motion, and while some level of compromise will be necessary due to practical limitations, the general approach adopted here is to start from standard validation cases in each area of relevance to the LGSA problem and then to work towards a more realistic simulation of the shock absorber system, adding multi-physics effects either in the form of a single integrated solver or as an iterative approach that takes account of these effects incrementally. Having area specific 
benchmarking cases will allow a better understanding of the solver's core capabilities and help identify the source of the errors observed in more complex multi-physics simulations. In addition, it is proposed to use a dynamic system model to generate physically consistent boundary conditions for the shock absorber moving walls, and to compare the general trends observed.

\subsection{Fluid Dynamic Field Solver}

OpenFOAM is used as the main fluid solver in all the validation cases presented, except the drop tests which are used to validate the dynamic system model. It is an open source solver, with a collection of $\mathrm{C}++$ libraries implementing an unstructured finite volume approach that is well suited for handling complex geometries and provides a wide range of numerical schemes and solution algorithms [2,3]. All simulations are conducted with second order accurate schemes.

\subsection{Dynamic System Model}

The dynamic system two mass model used in the present study follows the analysis of Milwitzky and Cook [4]. The equations are derived by applying Newton's second law of motion to the LGSA system with two degrees of freedom; the first associated with the main mass of the aircraft, while the second is related to the unsprung mass. The landing (or drop test) impact is split into two phases; initially the shock absorber acts as a rigid body until the impact force equals the internal preloading pneumatic pressure and friction forces, subsequently, it starts compressing with the full two degrees of freedom equations describing its motion.

3 Fluid Field Solver

3.1 Mixing Layer
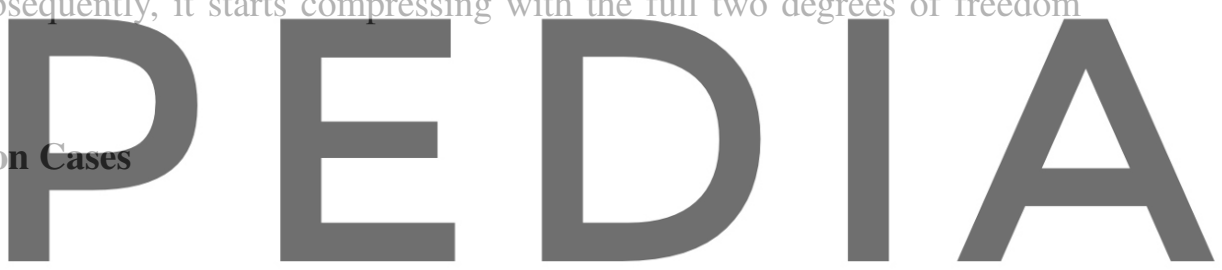

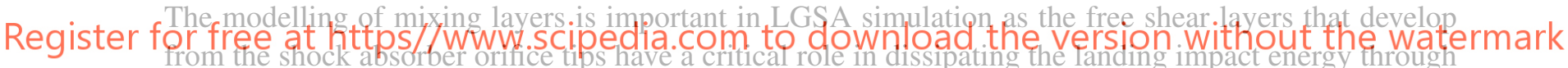
the turbulent scales generated that cascade the energy from the larger scales, which receive energy from the mean velocity gradient terms, to the smallest scales, where energy is dissipated as viscous heat.

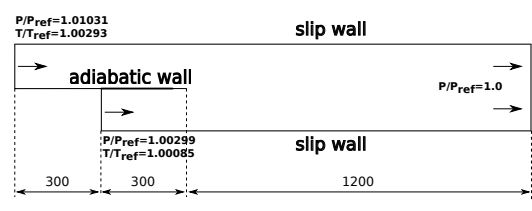

(a) Mixing Layer NASA Layout

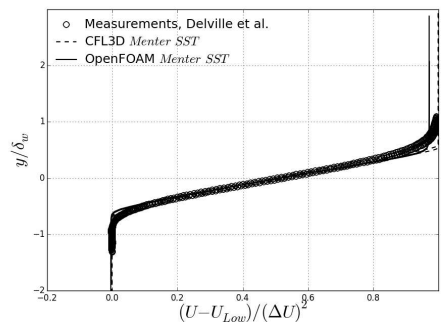

(b) Streamwise Velocity

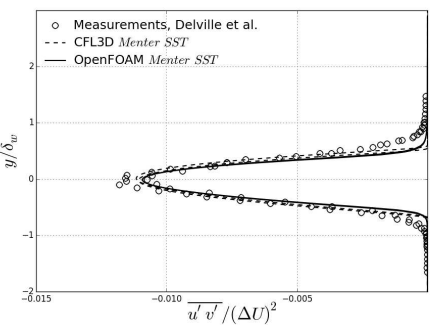

(c) Turbulent Shear Stress

Figure 1: Mixing Layer 
stream mixing layer with a sharp tip splitter plate separating them, where the setup is consistent with the experimental measurements by Delville et. al. [5]. The expected tendency towards self-similarity is captured in Fig. $1 \mathrm{~b}$ for the mean streamwise velocity and Fig. 1c for the Reynolds shear stress, $\overline{u^{\prime} v^{\prime}}$. The turbulent stresses need a longer streamwise development distance to reach self-similarity, which is consistent with previous mixing layer observations. The profile nearest the splitter plate tip shows the most discrepancy from the self-similar profile in the experimental measurements, while the RANS simulation tends to overreact to the presence of a mean velocity discontinuous profile, generating a fully developed turbulent flow sooner that is physically observed in experiments. This is not surprising because RANS models do not resolve or model the different stages of instabilities that lead to fully developed turbulent flows, but rather simply focus on the mean flow velocity gradients associated with the turbulence production terms in transport equations.

\subsection{Turbulent Boundary Layer}

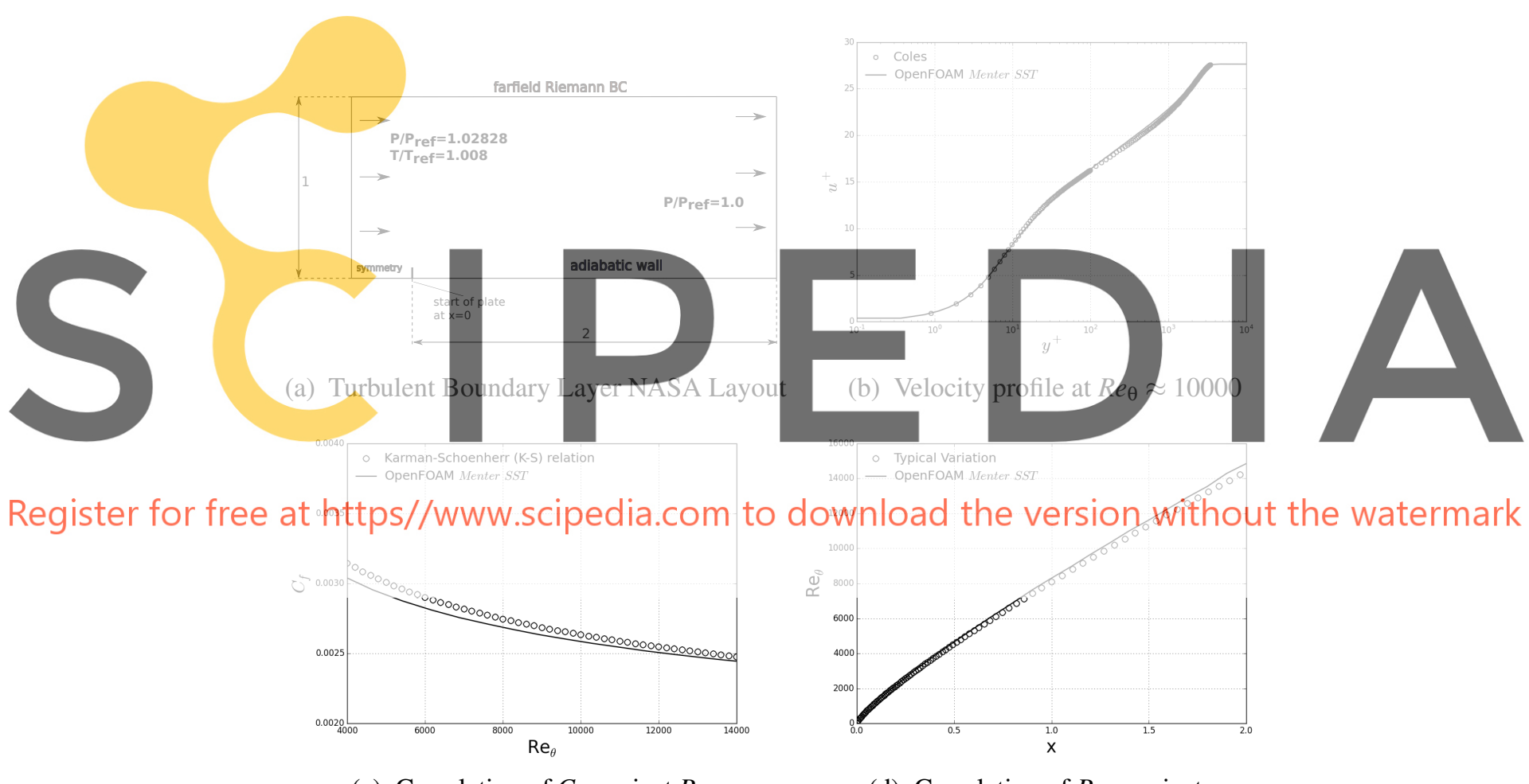

(c) Correlation of $C_{f}$ against $\operatorname{Re}_{\theta}$

(d) Correlation of $\operatorname{Re}_{\theta}$ against $x$

Figure 2: Turbulent Boundary Layer

Wall bounded turbulent flow is of relevance to the internal flow of LGSA where the high density hydraulic oil flowing against surfaces is expected to be in the turbulent regime for at least some stages of the motion. Predicting the skin friction and resistance to motion, as well as any separation that might take place are all important in understanding the internal dynamics.

The zero pressure gradient flat plate turbulent boundary layer is used to validate the standard near wall 
RANS model behaviour. The case is detailed in the NASA Turbulence Modeling Resource, and is illustrated in Fig. 3a. It is an important check when considering a hybrid LES-RANS approach, that models the near wall regions with RANS to alleviate the stringent grid requirements of wall resolved LES. The Log Law of the Wall in the fully turbulent region is correctly captured in agreement with the measurements of Coles [6], as shown by the velocity profile at $R e_{\Theta}=10000$ in Fig. 2b. The growth of the momentum thickness based Reynolds number, $R_{\Theta}$, is also well predicted in Fig. $2 \mathrm{c}$, and the skin friction correlation with $\operatorname{Re}_{\Theta}$, in Fig. 2d, is within the range of theoretical behaviour spread, where only the Karman-Shoenherr correlation is plotted in Fig. $2 d$ to reduce clutter.

\subsection{Separated Axisymmetric Boundary Layer}

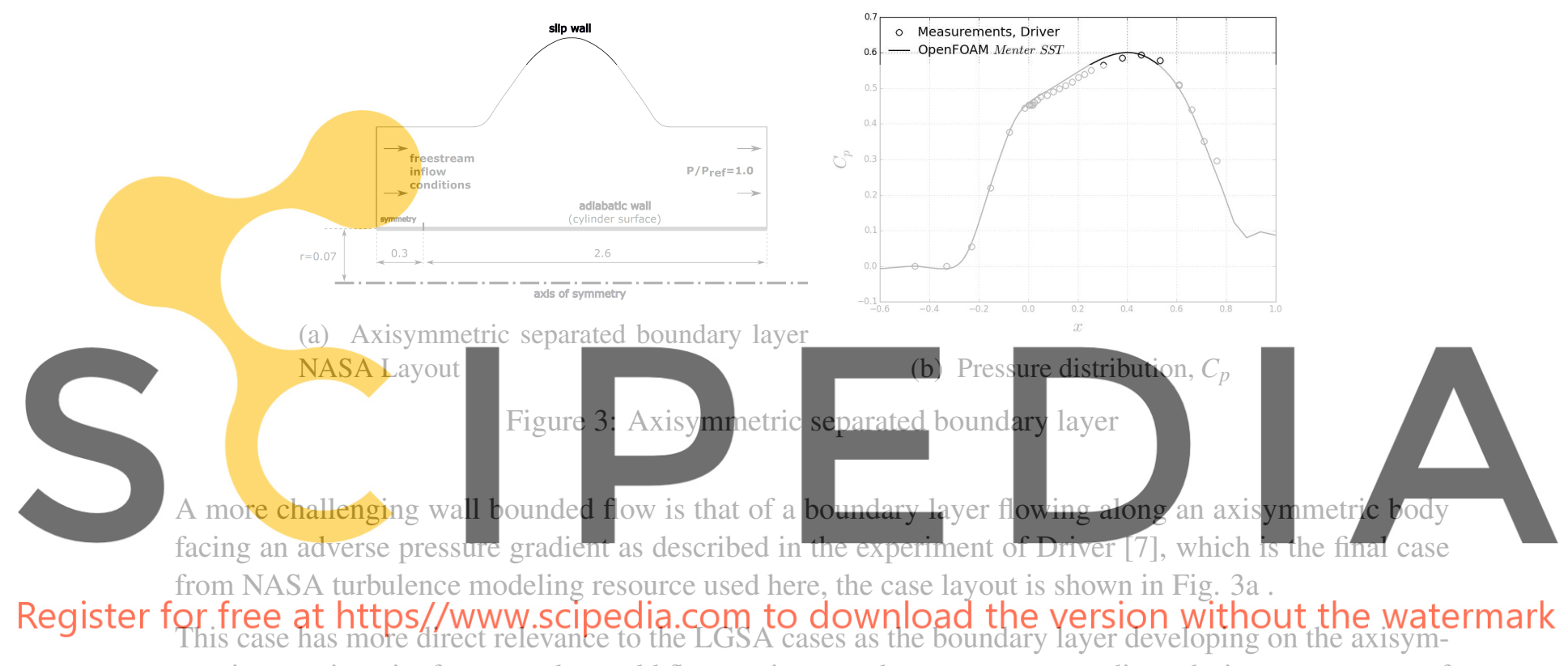

metric metering pin, for example, could flow against an adverse pressure gradient, during some stages of

the shock absorber motion, the pressure gradient imposed by the experimental configuration is correctly captured by the RANS model in Fig. 3b. The mean streamwise velocity profiles in Fig. 4 are initially turbulent and attached as the boundary layer approaches the adverse pressure gradient region, where it is decelerated until it separates forming a separation bubble and then reattaches further downstream. The velocity profiles in Fig. 4 are well predicted by the RANS model, while the Reynolds shear stress, $\overline{v^{\prime} u^{\prime}}$, profiles in Fig. 5 show some discrepancy in the maximum amplitude around the separation region, where strongly unsteady flow dominates. However, the near wall gradient of the turbulence shear stress profiles is well predicted throughout.

\subsection{Multiphase Flow: Rising Bubble}

Multiphase flow is relevant to oleo-pneumatic shock absorbers due to the presence of gas often in direct contact with hydraulic oil, leading to a range of potential interactions depending on the flow conditions. The rising bubble case is a standard benchmarking test for multiphase flow. The case setup is described 

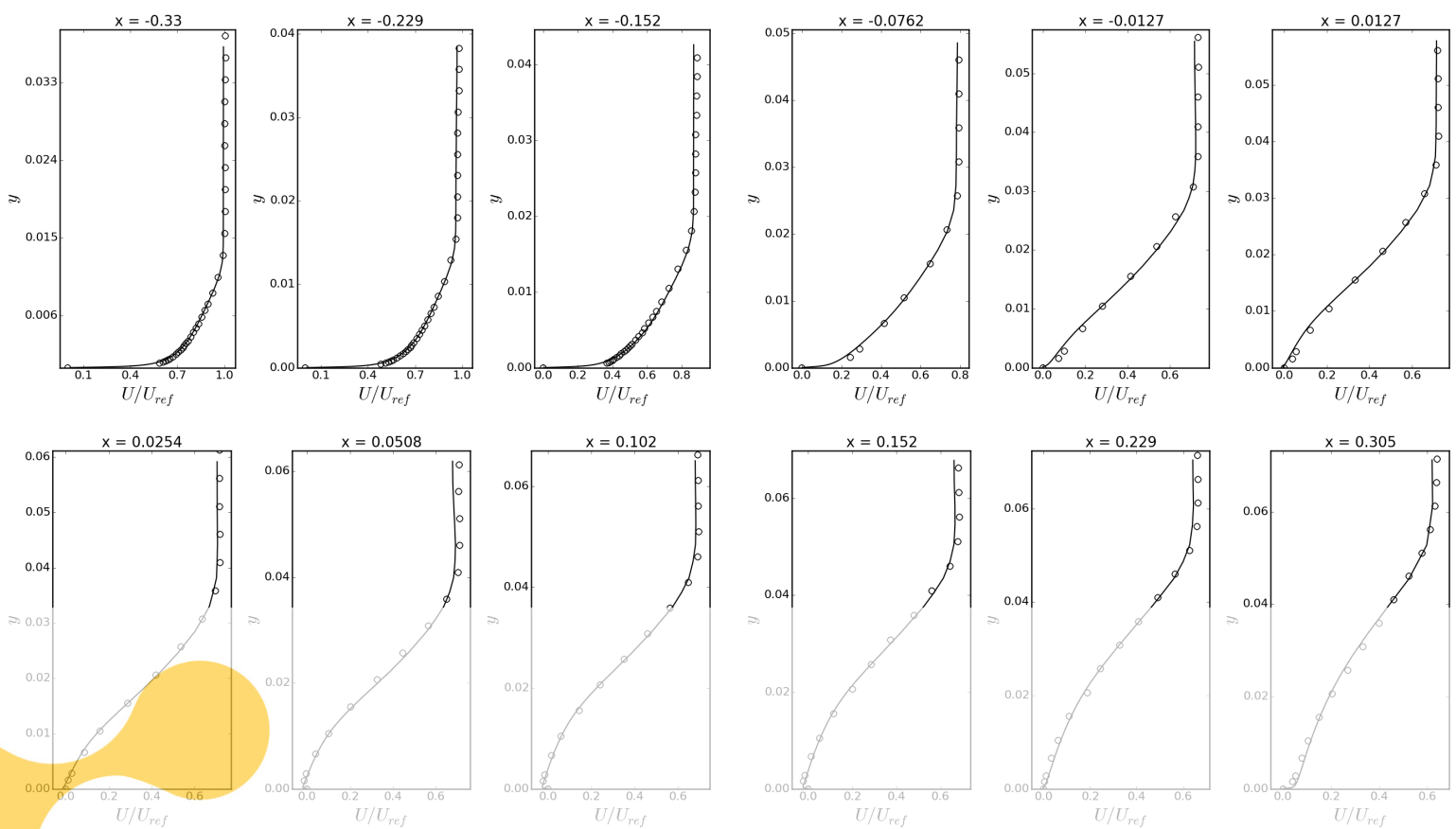

Figure 4: Separated boundary layer velocity profiles (Legend in Fig. 5)
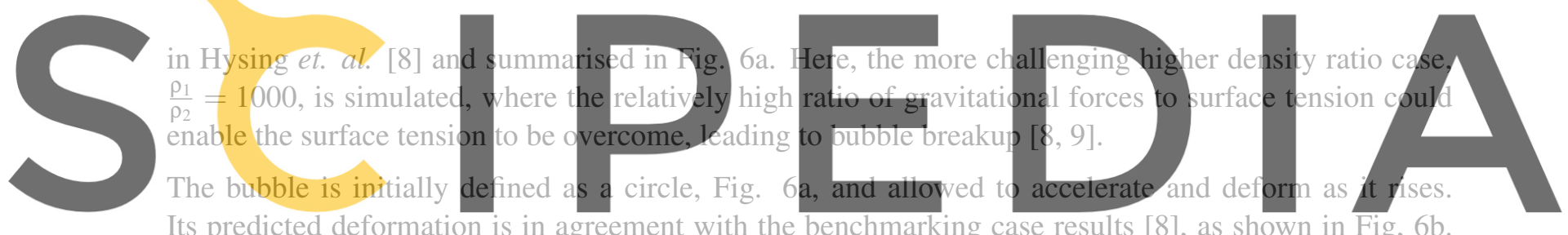

Its predicted deformation is in agreement with the benchmarking case results [8], as shown in Fig, 6b.

Register for free at https, www scipedia.com to download the version, without the watermark solvers used in the benchmarking study [8].

\subsection{Isentropic Compression}

Isentropic compression, Fig. 8a, is an idealised test case that is governed by $p V^{\gamma}=$ Const. It is used to test the dynamic mesh capability of the fluid solver. The pressure and temperature variation with volume are plotted in Figs. 8b and 8c respectively, where the simulations results are in almost perfect agreement with the analytical law. The compression of the gas spring in the shock absorber is expected to obey a polytropic law, $p V^{n}=$ Const, where the exponent $n$ is found from experimental measurements or high fidelity simulations.

\subsection{Conjugate Heat Transfer}

Heat transfer is another aspect of the multi-physics present in the LGSA problem that could influence the shock absorber performance due to variations in the fluid properties as a consequence of heat transfer. 

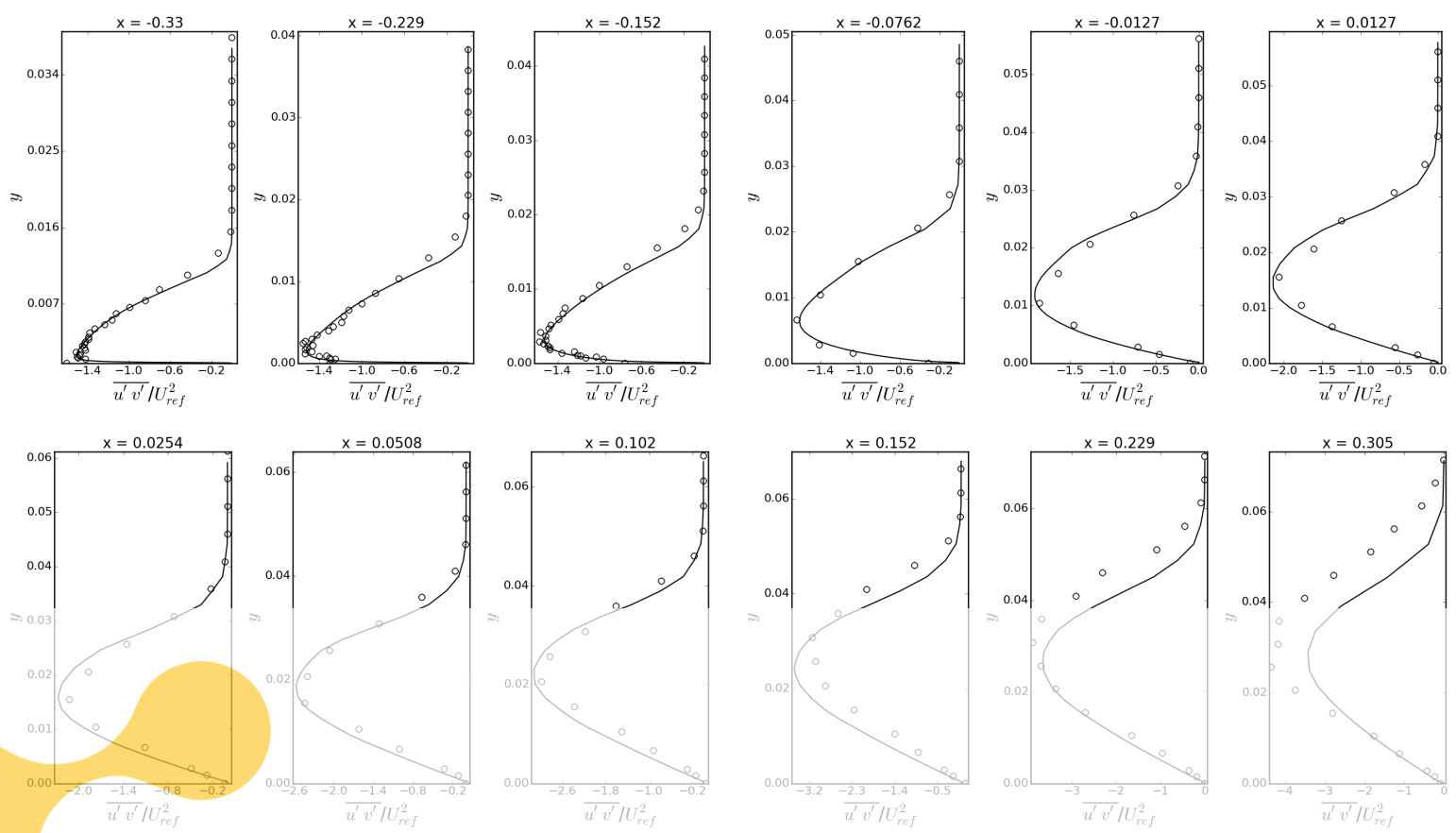

- Measurements, Driver — OpenFOAM Menter SST
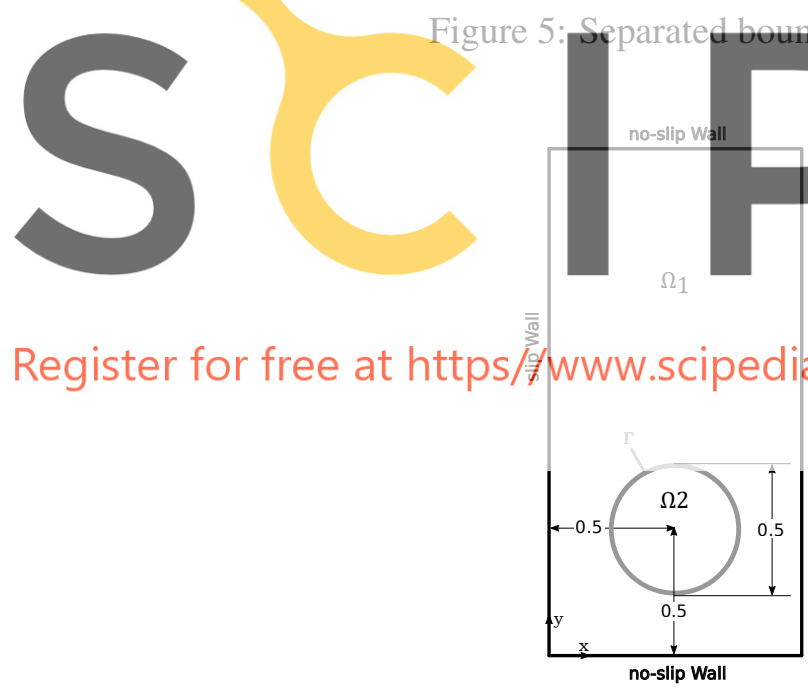

(a) Case setup
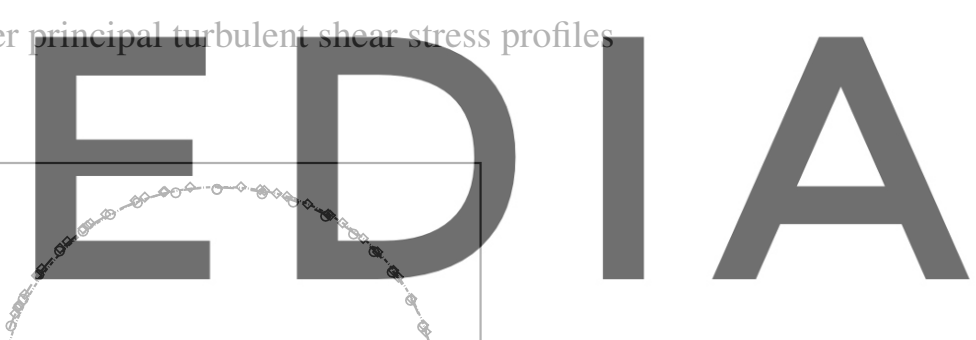

Register for free at https/部www.scipedila.com to download the version without the watermark

Figure 6: Bubble shape visualisation

During aircraft landing, the metal casing enclosing the LGSA can heat up quicker than the hydraulic oil in the chamber due to higher heat conductivity, resulting in potentially complicated gas-liquid-solid heat interaction among metal casing, bearing oil, chamber gas and hydraulic oil, and outside environmental air. The extent of this influence and its overall significance has to be determined from high fidelity multi-physics simulations, in the absence of any experimental data about these effects. 


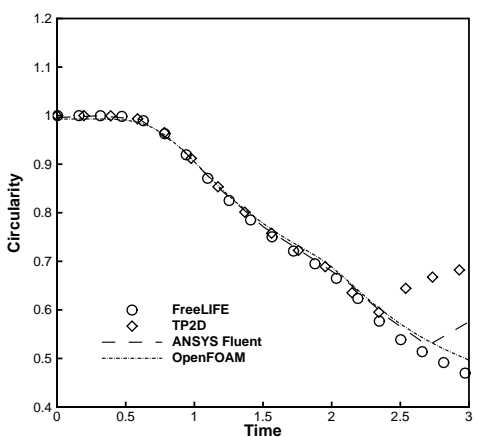

(a) Circularity

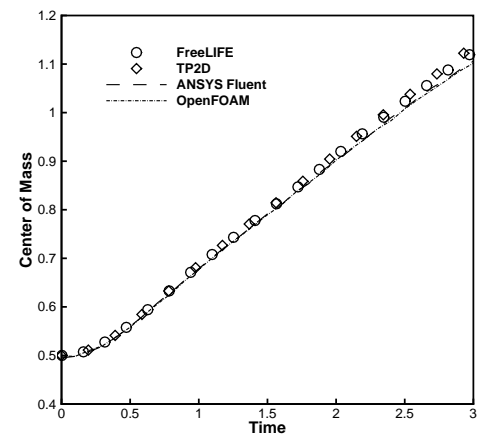

(b) Centre of Mass

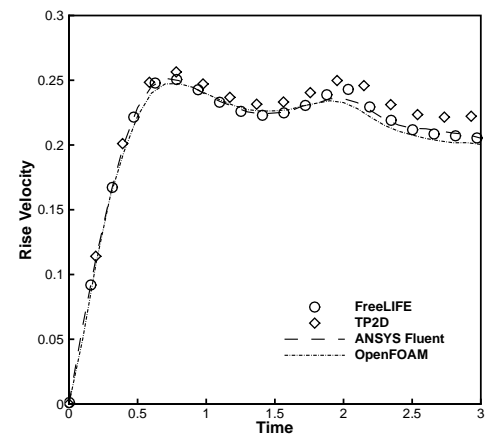

(c) Rise velocity

Figure 7: Rising bubble quantitative values
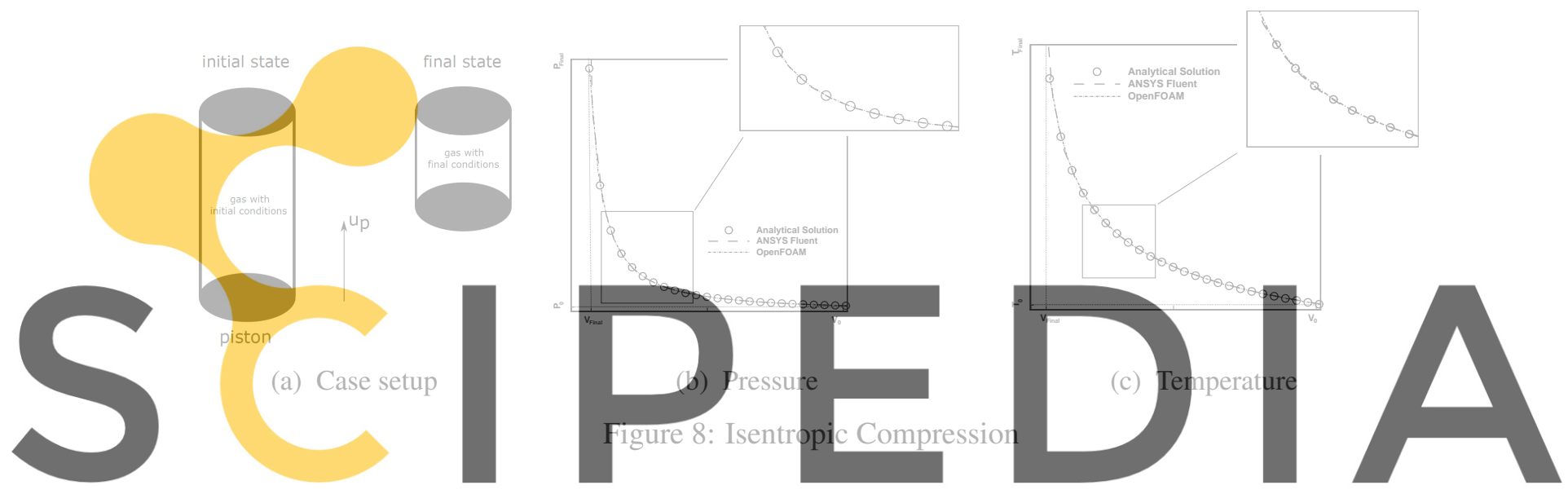

Register for free at https//www.scipedia.com to downfougd the version without the watermark

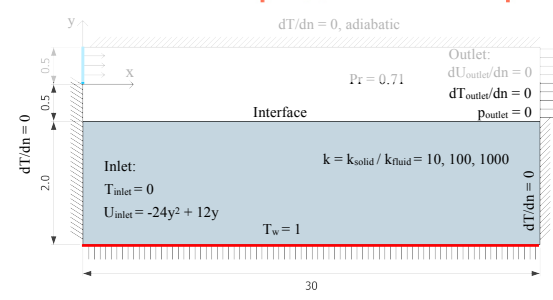

(a) Case setup

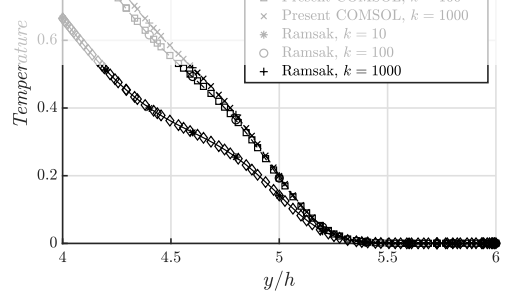

(b) Temperature variation in fluid

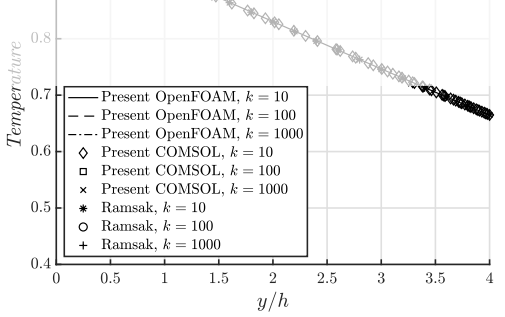

(c) Temperature variation in solid

Figure 9: Conjugate Heat Transfer at $x=6 h$

Conjugate heat transfer refers to the thermal interaction between a fluid and solid due to a temperature gradient between them. In the LGSA context, the temperature gradient could be generated due to bearing friction in the solid or due to gas compression in the fluid part of the shock absorber system.

The validation case of backward-facing step is setup following [10] as shown in Fig. 9a. It is also 
an attractive benchmarking case because it includes both conduction and convection dominated heat transfer. While there is a wealth of results available for this case, the temperature profiles from only one streamwise location, at $x=6 h$, are presented here, as all the other results match the reference data equally well. The temperature variation in the fluid in Fig. $9 \mathrm{~b}$ is non-linear as expected for a convection dominated heat transfer process. It is also worth noting that this streamwise location is near the unsteady recirculation region downstream of the step at inlet. The temperature variation in the solid, on the other hand, is dominated by conduction as demonstrated by the linear temperature variation in Fig. 9c.

\subsection{Natural Convection}

Natural convection is a buoyancy driven flow resulting from thermal interaction in the presence of a temperature gradient. It could have an influence on the equilibrium state of the LGSA system after the completion of a ground manoeuvre, such as a landing or rejected take-off, or after a change of state such as gear extension or retraction. Buoyancy driven flow is usually associated with a relatively low velocity and low heat transfer coefficient values, hence it is not expected to dominate the behaviour during the highly dynamic landing impact for example, but could affect the properties of the system after it has undergone some thermal variations. Its overall impact on LGSA performance, under different conditions, requires further testing.

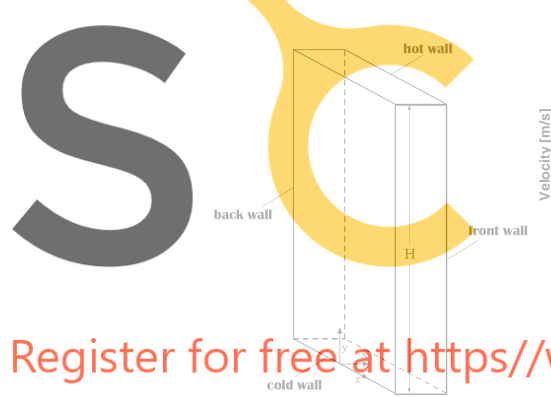

(a) Case setup

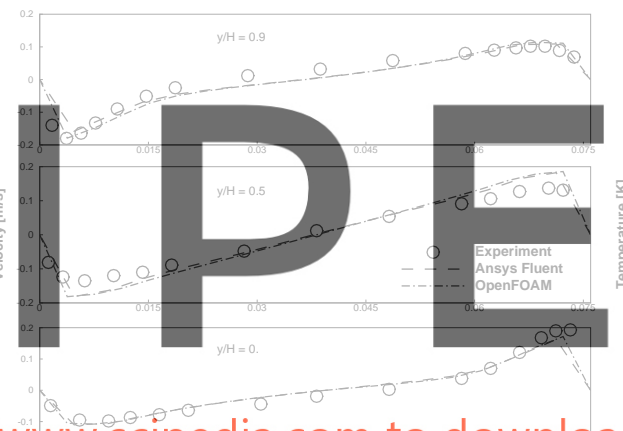

(b) Velocity

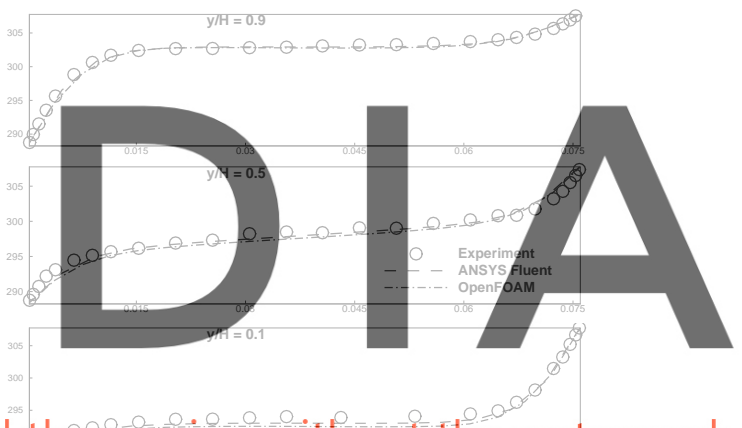

the version without whe watermark

(c) Temperature

Figure 10: Natural Heat Convection

The validation case is from Betts and Bokhari [11] focusing on the flow and thermal development in the gap between a hot and cold plates, as shown in Fig. 10a. The velocity and temperature profiles in the gaps at different heights are plotted in Figs. 10b and 10c respectively, where $y$ is here the streamwise direction from down up along the gap between the plates. The numerical results produced using OpenFOAM are generally in good agreement with the experimental measurements and captures the correct flow behaviour of rising fluid near the hot plate due to the reduced fluid density and sinking fluid near the cold plate, where the density is higher. 


\section{Dynamic System Model Validation}

\subsection{Response to Disturbance Functions}
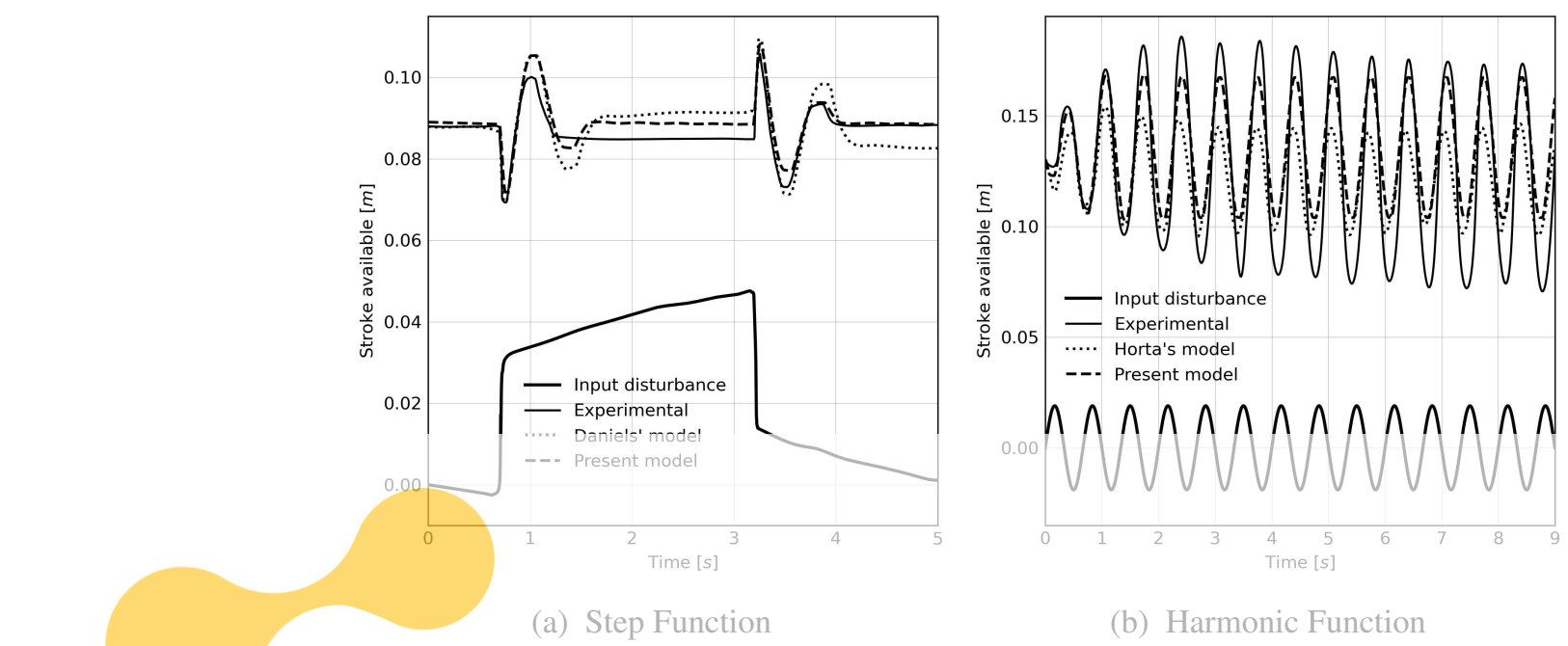

(b) Harmonic Function

Figure 11: Dynamic system response under specified forcing functions
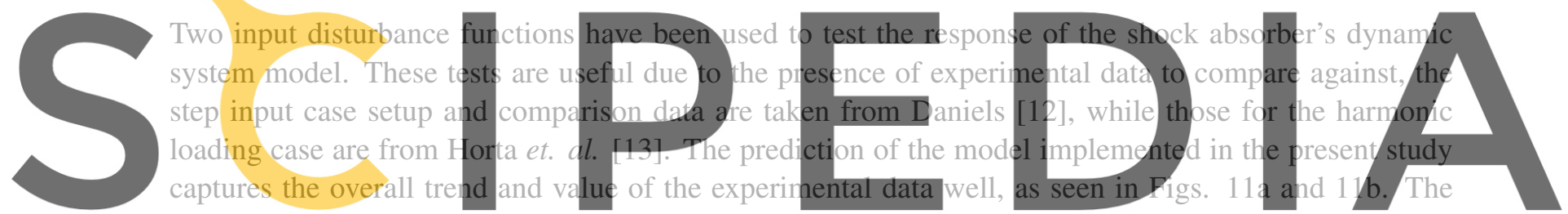

tendency to over or undershoot the peak experimental values is expected in regions of sharp gradients

Register for free at https//WWW.Scipedia.com to download the version without the watermark

\subsection{Drop Test}

The drop test details and the experimental measurements are available from Milwitzky and Cook [4]. The test here doesn't include tire bottoming, which simplifies the modelling of the vertical tire force. The two mass model prediction is compared against the experimental measurements in Fig. 12. The overall performance is promising with the system behaviour captured relatively well during the initial stages of the impact and during the compression phase, while the rebound (or unloading) part of the stroke is less accurately predicted. This could be due to a number of assumptions used in the derivation of the model being more appropriate during the initial compression phase. For example, the discharge coefficient assumes a fully turbulent and pure (single phase) fluid with a constant value used throughout the simulation, whereas the conditions inside a real world shock absorber are likely to result in some dependency on the stroke velocity and the fluid state, with possible foaming or aeration taking place due to multiphase effects. In addition, the fluid is assumed to impulsively adopt uniformly the relative velocity of the stroke and the analysis ignores the impact of variations in the distance upstream of the orifice available to the fluid, and it also ignores the effect of the no-slip boundary condition on the fluid in 


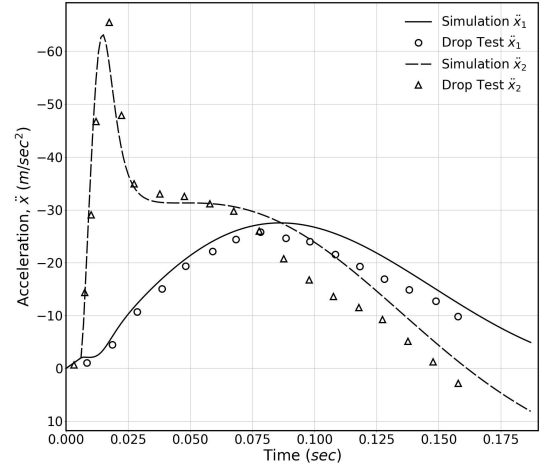

(a) Acceleration
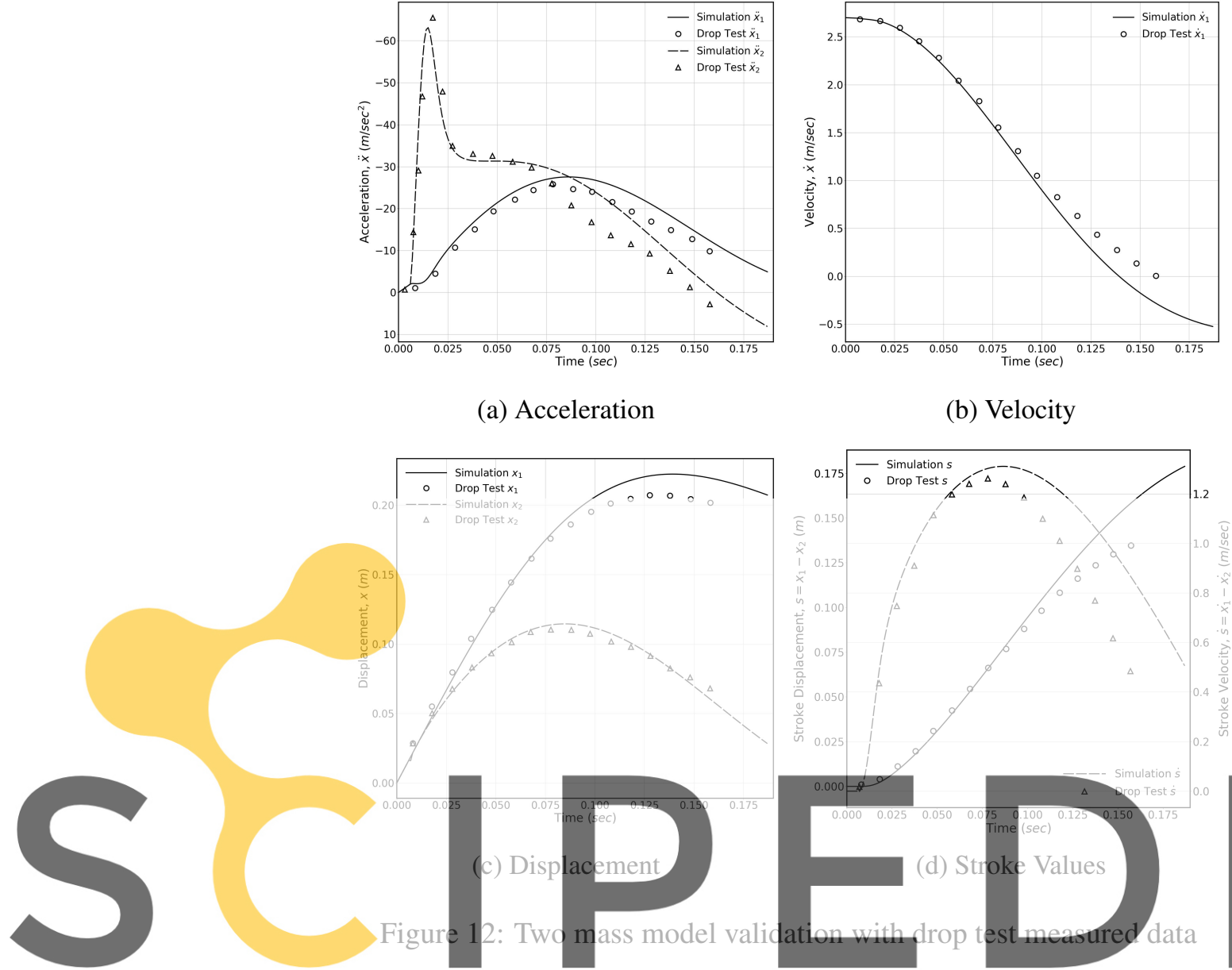

(b) Velocity

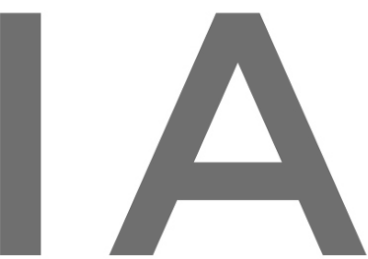

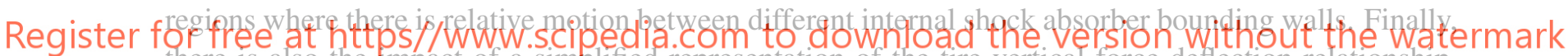
there is also the impact of a simplified representation of the tire vertical force deflection relationship,

which is more accurate in the compression regime, as implemented in the current version of the model.

\section{Steady Reynolds Averaged Navier-Stokes (RANS) Simulations}

The purpose of these preliminary RANS simulations is to gain an insight into the flow field development and identify the regions that would benefit from a higher grid density to resolve the turbulent scales in LES and hybrid LES-RANS computations. The simulation presented is 2D axisymmetric conducted using the steady RANS Menter Shear Stress Transport SST model [14], with hydraulic oil as the working fluid $\rho_{\text {oil }}=869 \mathrm{~kg} / \mathrm{m}^{3}$, a uniform inlet velocity of $3.0 \mathrm{~m} / \mathrm{s}$ and low inlet turbulence intensity $T i \approx 0.1 \%$.

The flow field through the main orifice with metering pin fully extended is presented in Fig 13. The strong blockage effect of the metering pin geometry is clearly visible in the velocity contours, 13b, with the flow through the orifice deflected towards the casing and creating a recirculation region downstream of the orifice. The turbulence kinetic energy contour plot, shown in Fig. 13c, clearly shows the development of two shear layers, one originating from the orifice free shear layer while the other developing from the 
boundary layer separating from the metering pin surface and interacting with the deflected flow. The two shear layers impinge on each other and the cylindrical casing of the shock absorber, with implications for the wall forces and energy dissipation.

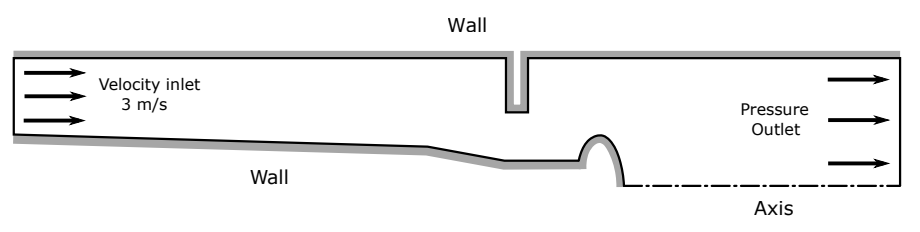

(a) Computational domain and boundary conditions for main orifice

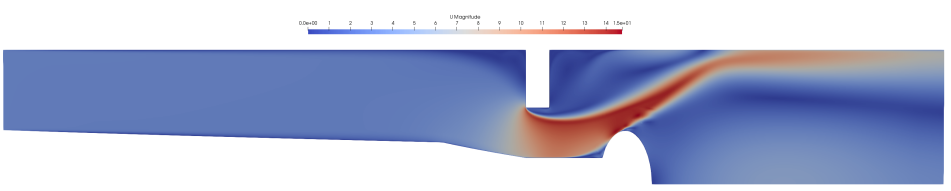

(b) Velocity Magnitude

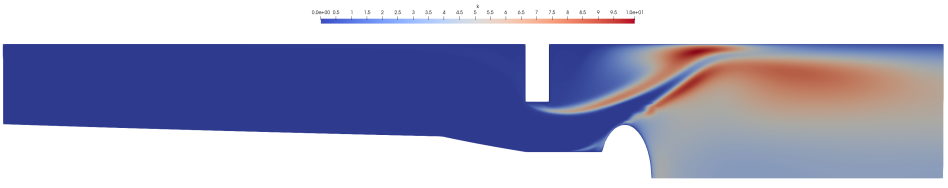

(c) Turbulence kinetic energy

Figure 13: Main Orifice with fully extended pin

The full picture is more complicated due to the presence of the gas spring on top of the hydraulic fluid, hence the deflected shear layers could reduce the multiphase interaction between the gas and oil. Conversely, the transient forces on the walls due to this side (radial) deflection might reduce the fatigue life of the walls, seals or bearings. High-fidelity unsteady simulations will be used to assess the relative merit of these competing effects. Admittedly, the flow presented in Fig. 13 is not necessarily fully representative of the flow in an actual shock absorber, where the incoming flow is unlikely to be uniform or laminar. Nonetheless, conducting steady RANS simulations at different stroke positions for the compression and rebound motion can be extremely useful in the design of high fidelity cases, which in turn could be used to derive more representative boundary conditions for RANS.

\section{Conclusions}

The validation process and case setup for a multi-physics high fidelity oleo-pneumatic shock absorber simulation were discussed. The validation cases were chosen to cover a range of flow physics relevant to shock absorber, thus providing a benchmark for the expected solver behaviour in each case. A dynamic system model was also validated and used to generate boundary conditions for the moving walls in high fidelity simulations, while RANS simulations provided an insight into the expected flow development, and will be used in designing the higher fidelity cases. Future work will focus on conducting high fidelity multi-physics simulations and the corresponding data analysis. 


\section{Acknowledgement}

This work was supported by Innovate UK [Grant Number 263261]; and Airbus UK. The underlying data can be accessed at https://doi.org/10.17862/cranfield.rd.13865042, for the data appearing in the figures. Raw data can be made available on request.

\section{REFERENCES}

[1] N. Currey, Aircraft Landing Gear Design: Principles and Practices. AIAA education series, American Institute of Aeronautics and Astronautics, 1988.

[2] H. G. Weller, G. Tabor, H. Jasak, and C. Fureby, "A tensorial approach to computational continuum mechanics using object-oriented techniques," Computers in physics, vol. 12, no. 6, pp. 620-631, 1998.

[3] H. Jasak, Error analysis and estimation for the finite volume method with applications to fluid flows. $\mathrm{PhD}$ thesis, Imperial College London, 1996.

[4] B. Milwitzky and F. E. Cook, "Analysis of landing-gear behavior," NACA Technical Note, no. TN$2755,1952$.

[5] J. Delville, S. Bellin, J. Garem, and J. Bonnet, "Analysis of structures in a turbulent, plane mixing layer by use of a pseudo flow visualization method based on hot-wire anemometry," in Advances in Turbulence 2, pp. 251-256, Springer, 1989.

[6] D. Coles, "The law of the wake in the turbulent boundary layer," Journal of Fluid Mechanics, vol. 1, no. 2, pp. 191-226, 1956.

[7] D. DRIVER, "Reynolds shear stress measurements in a separated boundary layer flow," in 22nd Fluid Dynamics, Plasma Dynamics and Lasers Conference, no. AIAA Paper 91-1787, 1991.

[8] S.-R. Hysing, S. Turek, D. Kuzmin, N. Parolini, E. Burman, S. Ganesan, and L. Tobiska, "Quantitative benchmark computations of two-dimensional bubble dynamics," International Journal for Numerical Methods in Fluids, vol. 60, no. 11, pp. 1259-1288, 2009.

[9] R. Clift, J. R. Grace, and M. E. Weber, "Bubbles, drops, and particles," 2005.

[10] M. Ramšak, "Conjugate heat transfer of backward-facing step flow: A benchmark problem revisited," International Journal of Heat and Mass Transfer, vol. 84, pp. 791-799, 2015.

[11] P. Betts and I. Bokhari, "Experiments on turbulent natural convection in an enclosed tall cavity," International Journal of Heat and Fluid Flow, vol. 21, no. 6, pp. 675-683, 2000.

[12] J. N. Daniels, "A method for landing gear modeling and simulation with experimental validation," NASA Contractor Report R-1154, no. CR-201601, 1996.

[13] L. G. Horta, R. H. Daugherty, and V. J. Martinson, "Modeling and validation of a navy a6-intruder actively controlled landing gear system," NASA Technical Publication, no. TP-1999-209124, 1999.

[14] F. R. Menter, "Two-equation eddy-viscosity turbulence models for engineering applications," AIAA journal, vol. 32, no. 8, pp. 1598-1605, 1994. 